Perspective

\title{
On cancer electro- and nano-chemotherapy
}

Volume 7 Issue 2 - 2017

\author{
Abbreviations: BBB, blood brain barrier; ECT, \\ electrochemotherapy; MRSA, methycillin-resistant staphylococcus \\ aureus; NCT, nanochemotherapy; OPN, osteopontin
}

\section{Diseases listed-cancer types}

Adenocacinoma, Basal cell carcinoma, Breast cancer, Kaposi sarcoma, Melanoma, Squamous cell carcinoma.

\section{Drugs cited}

Accurin, Bleomycin, Cisplatin, Osteopontin.

\section{Introduction}

Chemotherapy (or anti-cancer drug therapy) is a systemic drug treatment in which chemotherapeutic drugs are imparted to the blood to circulate through all parts of the body. Its purpose is to control or kill the cancer. A testimony to its effectiveness is the increasing number of cancer survivors. Most chemotherapy drugs are delivered by different systems through a vein (IV), injected into a muscle, under the skin, into spaces around body tissues, directly into cancerous masses or swallowed (pill), and even intranasally for direct access to brain cancers but not all drugs are available in all delivery forms. Whereas I have described in earlier articles recent research developments in anti-cancer therapy ${ }^{1}$ and in Nanomedicine, ${ }^{2,3} \mathrm{I}$ am here interested in two important types of chemotherapy delivery systems that could more efficiently deliver the drugs to the tumor sites, specifically electrochemotherapy and nanochemotherapy.

As a preamble, Table 1 lists the more common tumor markers or substances that can indicate the presence of specific types of cancer and can also be used to assess the treatment efficacy: On the other hand, Table 2 summarizes some of the more than 50 different chemotherapy drugs within six broad categories. Note that some cancers can be treated with drugs of different categories either singly or in combination (last column of Table 2) ${ }^{4}$

Table I List of the more common tumor markers

\begin{tabular}{ll}
\hline Antigens & Others \\
\hline Carcinoembryonic & Human chorionic gonadotropic \\
Carbohydrate & Alpha-fetoprotein \\
& 5-Hydroxy indole acetic acid \\
Prostate-specific & $\begin{array}{l}\text { Serum paraproteins } \\
\text { Nucleic acids }\end{array}$ \\
\hline
\end{tabular}

\section{Electrochemotherapy}

Electrochemotherapy (ECT) delivers non-permeant drugs to the cell interior. The technique is based on the local application of short and intense electric pulses that transiently permeabilize the cell membrane, thus allowing the transport of chemotherapeutic molecules through it.

\section{Physical principle}

When a biological cell is exposed to an electric field of sufficient strength, the voltage across the membrane is increased leading to (a)

\author{
Alain L Fymat \\ International Institute of Medicine and Science, USA \\ Correspondence: Alain L Fymat, International Institute of \\ Medicine and Science, California, USA,, Tel (760) 485-9149, \\ Email alain.fymat@fiimas.org \\ Received: December 21, 2016 | Published: January 20, 2017
}

rearrangements of its structure and (b) an increase of its permeability, allowing nonpermeant molecules to enter the cell (a phenomenon called electroporation or electropermeabilization). The approach is becoming widely used to improve anticancer drug delivery into cells (from which the name electrochemotherapy). The pulse characteristics (amplitude, phase, pulse duration and repetition frequency, DCor AC-currents, shape and position of the electrodes) are chosen depending on the tissues; however, the pulse amplitude has to be high enough to establish a $400 \mathrm{Volt} / \mathrm{cm}$ electrical field in the tumor area and yield eight $100 \mathrm{~ms}$ pulses at a $5000 \mathrm{~Hz}$ repetition frequency for patient comfort and shorter treatment duration. ${ }^{6-8}$ For deep-seated tumors in the relative vicinity of the heart, pulses are synchronized with the absolute refractory period of each heart beat so as to minimize the probability of their interaction with the heart function.

\section{Treatment protocol and safety}

The ECT treatment consists in applying electric pulses to the area to be treated and delivering, either systemically or locally, non-permeant cytotoxic drugs (e.g., bleomycin) or low-permeant drugs (e.g., cisplatin, an alkalyting agent, Table 2). The pulses cause nanoscale defects on the cell membrane, which alter its permeability. The drugs can then freely diffuse into the cytoplasm and exert their cytotoxic effect. As in radiotherapy where high energy radiation is imparted through multiple ports and at different times (so-called dose and time fractionation), multiple electrodes positions associated with a sufficient drug concentration and repeated at subsequent time intervals can likewise be used to treat the whole tumor. ${ }^{9-12}$

\section{Efficacy and clinical relevance}

ECT has been widely used for cutaneous and subcutaneous tumors or their metastases, yielding an objective response rate $>80 \%$ and, when compared to standard chemotherapy, a faster and more efficient 
tumor size reduction. Patients with skin metastasis from melanoma, Kaposi sarcoma, squamous cell carcinoma, basal cell carcinoma, adenocarcinoma, or breast cancer have been successfully treated. First clinical results for internal tumors (e.g., liver metastases) and their treatment using surgical procedures, endoscopic routes, or percutaneous approaches to gain access to the treatment area have been promising and encouraging. Began in the early 1990s, such treatments are now provided across the world. ${ }^{13}$

Table 2 Broad classification of the different chemotherapeutic drugs ${ }^{5}$

\begin{tabular}{|c|c|c|c|}
\hline $\begin{array}{l}\text { Drug } \\
\text { Category }\end{array}$ & Drug Names & $\operatorname{Aim}(\mathbf{s})$ & Cancer Types \\
\hline \multirow{2}{*}{$\begin{array}{l}\text { Antibodies } \\
\text { ("magic bullets) }\end{array}$} & $\begin{array}{l}\text { Bevacizumab, Cetuximab, Erlotinib*, } \\
\text { Getifinib*, Imatinib*, Rituximab*, } \\
\text { Trastuzumab* }\end{array}$ & Cancer cells, sparing normal cells & \multirow[t]{2}{*}{$\begin{array}{l}\text { Breast, colon, lung, lymphoma, several types } \\
\text { of leukemia }\end{array}$} \\
\hline & $\begin{array}{l}\text { *also used in biological } \\
\text { therapy that helps the immune } \\
\text { system destroy the cancer cells }\end{array}$ & & \\
\hline $\begin{array}{l}\text { Alkylating } \\
\text { agents }\end{array}$ & $\begin{array}{l}\text { Busulfan, Carboplatin, Chlorambucil, } \\
\text { Cisplatin, Cyclophosphamide, Thiotepa }\end{array}$ & Cell's DNA (to prevent further cell division) & $\begin{array}{l}\text { Chronic leukemia, non-Hodgkin's lymphoma, } \\
\text { Hodgkin disease, multiple myeloma, lung, } \\
\text { breast, ovary }\end{array}$ \\
\hline Nitrosoureas & Carmustine, Lomustine & Interfere with enzymes that help repair DNA & $\begin{array}{l}\text { Brain, non-Hodgkin lymphoma, multiple } \\
\text { myeloma, malignant melanoma }\end{array}$ \\
\hline Antimetabolites & $\begin{array}{l}\text { Capeticabine, Cytarabine, Fludarabine, } \\
\text { r-Fluorouracil, Gemcitabine, } \\
\text { Methotrexate }\end{array}$ & $\begin{array}{l}\text { Alter enzyme functions in cell metabolism } \\
\text { and protein synthesis, starving the cells to } \\
\text { death }\end{array}$ & $\begin{array}{l}\text { Leukemia (acute, chronic), breast, ovary, } \\
\text { gastrointestinal tract }\end{array}$ \\
\hline $\begin{array}{l}\text { Antitumor } \\
\text { antibiotics }\end{array}$ & $\begin{array}{l}\text { Dactinomycin, Daunorubicin, } \\
\text { Doxorubicin, Epirubicin, Idarubicin, } \\
\text { Mitoxantrone }\end{array}$ & $\begin{array}{l}\text { Bind with DNA and prevent RNA synthesis } \\
\text { (imperative for cell survival) }\end{array}$ & Many different cancers \\
\hline $\begin{array}{l}\text { Miotic } \\
\text { inhibitors }\end{array}$ & $\begin{array}{l}\text { Docetaxel, Paclitaxel,Vinblastine, } \\
\text { Vincristine,Vinorelbine }\end{array}$ & $\begin{array}{l}\text { Inhibit cell division/ reproduction and use of } \\
\text { certain proteins required for mitosis }\end{array}$ & Leukemia, lymphomas, lung, breast \\
\hline
\end{tabular}

\section{Safety}

ECT employs lower dosages of chemotherapeutic drugs than standard chemotherapy protocols resulting in lower patient's burden and more limited side effects. Further, local anesthesia can alleviate muscle contraction and pain due to the application of electric pulses. ECT treatments have been approved and reimbursed in several EU countries with CE-marked devices certified as medical devices offered on the market. Two noteworthy effects have been noted: (a) In healthy tissues: The types of pulses used by ECT induce a vascular block wherein, for a few minutes, blood flow is interrupted in the treated volume in the normal tissues. The pulse duration is too short to induce deleterious effects due to ischemia; and (b) in tumors: The vascular block is of a longer duration, contributing to the effectiveness of the ECT treatment.

\section{Nanochemotherapy}

Nanochemotherapy (NCT) uses nanodevices to deliver nanoparticles containing cytotoxic drugs to tumors. The basic process to use drug delivery here involves at least three steps: (a) Encapsulation of the drugs; (b) Successful delivery of said drugs to the targeted region of the body; and (c) Successful release of that drug there.

\section{Devices for drug delivery and therapeutics}

Engineered nanoscale devices are minute devices with the potential to be engineered to efficiently and more safely deliver drug treatments directly to the location of diseased cells while helping avoid harm to healthy cells that fall victim to toxic drugs administered by conventional means. A library has been developed of 800 different and uniquely shaped hybrid nanocrystals, formed from ordered atom clusters that act as new tools or molecular tags enabling and aiding targeted drug delivery. ${ }^{14}$ These new nanocrystals are multifunctional and able to be multi-tasked to do different things simultaneously. Their fabrication can be precisely controlled to create different shapes and sizes, allowing the assessment of the drug impact along its propagation path within the body.

Because of their diverse capabilities, nanoscale devices can contain both targeting and therapeutic agents (in both single and multi-drug approaches). They can deliver high drug levels in several situations, including anticancer drugs at the tumor site that can increase chemotherapeutic efficacy. They can also offer the opportunity to develop new approaches to therapy, including "smart" nanotherapeutics to "time" the release of any given drug or to deliver multiple drugs sequentially in a timed manner or at several locations in the body.

\section{Nanoparticles}

Several nanoparticles are employed.

\section{Nutshells ( $120 \mathrm{~nm}$ in diameter coated with gold)}

The nutshells can be targeted to bond to cancerous cells by conjugated antibodies or peptides to the anopheles' surface. By irradiating the tumor with an infra-red (IR) laser, which passes through flesh without heating it, the gold is heated sufficiently to cause death to the cancer cells.

\section{Platelet-coated nanoparticles ( $100 \mathrm{~nm}$ in diameter)}

They can deliver drugs to targeted sites in the body, particularly injured blood vessels, as well as organs infected by harmful bacteria. By delivering the drugs to the sites where they are needed, these nanoparticles can greatly increase their therapeutic effects by directly depositing a much higher dose of medication specifically to diseased 
areas without saturating the entire body with drugs. This principle has broad implications for targeted therapy for other diseases such as cancer and neurological disorders.

\section{Gelatin nanoparticles for delivering multiple drugs to the brain}

Gelatin is biocompatible, biodegradable, and classified as "generally recognized as safe" by the FDA. Once administered, the gelatin nanoparticles target damaged brain tissue thanks to an abundance of gelatin-munching enzymes produced in injured regions. They also have neuroprotective effects. Laced with the drug osteopontin (OPN), they can be administeed intranasally along the olfactory nerve cells-a noninvasive and direct route to the brain, to reduce inflammation and prevent brain cell death. This delivery pathway bypasses the blood brain barrier (BBB)-a biological fence that prevents the vast majority of drugs from entering the brain through the bloodstream, can be most effective in delivering drugs that cannot otherwise cross it, and can deliver therapeutics agents to specific regions of the brain. As far as is known, gelatin particles have not yet been used to treat glioblastomas.

\section{Shape-shifting engineered nanoparticles}

Nanoparticles can also be engineered so as to respond to biological molecules by changing shape to gain access to diseased tissue. These shape-shifters are made of minuscule chunks of metal with strands of DNA attached to them. This targeted molecular delivery system uses modular nanoparticles whose shape, size and chemistry can be altered by the presence of specific DNA sequences. The nanoparticles float around harmlessly in the blood stream until a DNA strand binds to a sequence of DNA known to be a marker for cancer. When this happens, the particle changes shape then carries out its function: target the cancer cells, expose a drug molecule to the cancerous cell, and tag the cancerous cells with a signal molecule. This approach can theoretically be imbedded in personalized nanomedical treatments, further tailoring the particles to deliver drugs to specified tumors and nowhere else.

\section{Kinase inhibitors in nanoparticle formulation}

Efforts to apply nanotechnology in cancer have focused almost exclusively on the delivery of cytotoxic drugs to improve therapeutic index. There has been little consideration of molecularly targeted agents, in particular kinase inhibitors, which can also present considerable therapeutic index limitations. Examples are accurin polymeric nanoparticles that encapsulate the clinical candidate AZD2811 (an Aurora B kinase inhibitor) using an ion-pairing approach. ${ }^{15}$ Accurins increase biodistribution to tumor sites, provide extended release of encapsulated drug payloads, show accumulation and retention in tumors with minimal impact on bone marrow pathology, and result in lower toxicity and increased efficacy. Accurins specifically, and nanotechnology in general, can increase the therapeutic index of molecularly targeted agents, including kinase inhibitors targeting cell cycle and oncogenic signal transduction pathways. A phase 1 clinical trial is the next step for this novel nanomedicine approach.

\section{Bioavailability-improved nanoscale particles and molecules}

Nanoscalea particles and molecules can also be developed to improve drug bioavailability, i.e., the presence of drug molecules where they are needed in the body and where they will do the most good. Drug delivery focuses on maximizing bioavailability both at specific places in the body and over a period of time. It can be achieved by employing nano-engineered devices that target the molecules and deliver drugs with cell precision.

\section{Clinical advantages}

There are several medical advantages to nanoparticles, including:

A. They circulate throughout the bloodstream without being attacked by the immune system.

B. They preferentially bind to damaged blood vessels and certain pathogens such as MRSA (Methycillin Resistant Staphylococcus Aureus) bacteria, allowing them to deliver and release their drug payloads specifically to these body sites in the body.

C. They are non-toxic as the platelet membranes are nanoparticle cores made of a biodegradable polymer that can be safely metabolized by the body.

They can be packed with many small drug molecules that diffuse out of the polymer core and through the platelet membrane onto their targets.

\section{Differential response to the same cancer treatment}

Tracking the path of chemotherapy drugs in real time and at the cellular level could revolutionize cancer care and help sort out why two patients might respond differently to the same treatment. Up until now, this was accomplished, admittedly in a limited way, by organic dyes (that faded quickly) and by toxic elements (particularly, metals). Recently, researchers at the Ohio State University have devised an organic technique using nanotechnology to light up a common cancer drug doxoribucin (Table 2, antitumor antibiotics) or any other cancer drug. They could see where the chemotherapeutic drug goes and how long it takes to get there. They first created a luminescent molecule (a peptide made of two amino acids) and hitched it to the cancer medication so that it revealed the drug pathway and arrival within the cells. ${ }^{16}$ Importantly, as it enters the cancerous site, that peptide easily coexists with human cells and leaves them harmless as it is composed of natural amino acids. Further, the nanoparticle is inherently biocompatible.

\section{Acknowledgments}

None.

\section{Conflicts of interest}

Author declares there are no conflicts of interest.

\section{References}

1. Fymat AL. Recent Research Developments in Anti-Cancer Therapy. $J$ Cancer Prev Curr Res. 2016;5(2):00155.

2. Fymat AL. Recent Developments in Nanomedicine Research. $J$ Nanomed Res. 2016;(4):00096.

3. Fymat AL. Nanotechnology and Cancer, $J$ Cancer Prev Curr Res. 2016;5(6):00180.

4. Fymat AL. The Long Quest for Cancer Cures. J Cancer Prev Curr Res. 2016;6(2):00201.

5. Lyss AP, Fagundes HM, Corrigan P. Chemotherapy and Radiation. Wiley Publishing Inc: New Jersey, USA; 2005. 388 p.

6. Mir LM, Orlowski S, Belehradek J, et al. Electrochemotherapy potentiation of antitumour effect of bleomycin by local electric pulses. Eur J Cancer. 1991;27(1):68-72. 
7. Hampton T. Electric pulses help with chemotherapy, may open new paths for other agents. JAMA. 2011;305(6):549-551.

8. Sersa G, Miklavcic D, Miklavcic. Electochemotherapy of tumours. $J$ Vis Exp. 2008;22.

9. Marty M, Sersa G, Garbay JR, et al. Electrochemotherapy-An easy, highly effective and safe treatment of cutaneous and subcutaneous metastases: Results of ESOPE (European Standard Operating Procedures of Electrochemotherapy) study. European Journal of Cancer Supplements. 2006;4(11):3-13.

10. Sersa G, Miklavcic D, Cemazar M, et al. Electrochemotherapy in treatment of tumors. Eur J Surg Oncol. 2008;34(2):232-240.

11. Mir LM, Belehradek M, Domenge C, et al. Electrochemotherapy, a new antitumor treatment: first clinical trial". CR Acad Sci III. 1991;313(13):613-618.
12. Miklavcic D, Snoj M, Zupanic A, et al. Towards treatment planning and treatment of deep-seated solid tumors by electrochemotherapy. Biomed eng online. 2010;9:10.

13. Rudolf Z, Stabuc B, Cemazar M, et al. Electrochemotherapy with bleomycin. The first clinical experience in malignant melanoma patients. Radiol Oncol. 1995;29(6).

14. Jin D, Deming L, Xiaxau X, et al. Three-dimensional controlled growth of sub-50 nm heterogeneous nanocrystals. Nature communications. 2015;7:10254

15. Ashton S, Song YH, Nolam J, et al . Aurora kinase inhibitor nanoparticles target tumors with favorable therapeutic index in vivo. Science Translational Medicine. 2016;8(325):325Ra17.

16. Zhang. Bioinspired Fluorescent Dipeptide Nanoparticles for Targeted Cancer Cell Imaging and Real-Time Monitoring of Drug Release, Nat Nanotech. 2016;11(4):388-394. 